\title{
Association of the infection probability of COVID-19 with ventilation rates in confined spaces
}

\author{
Hui Dai', Bin Zhao ${ }^{1,2}(\square)$ \\ 1. Department of Building Science, School of Architecture, Tsinghua University, Beijing 100084, China \\ 2. Beijing Key Laboratory of Indoor Air Quality Evaluation and Control, Tsinghua University, Beijing 100084, China
}

\begin{abstract}
A growing number of cases have proved the possibility of airborne transmission of the coronavirus disease 2019 (COVID-19). Ensuring an adequate ventilation rate is essential to reduce the risk of infection in confined spaces. In this study, we estimated the association between the infection probability and ventilation rates with the Wells-Riley equation, where the quantum generation rate $(q)$ by a COVID-19 infector was obtained using a reproductive number-based fitting approach. The estimated $q$ value of COVID-19 is $14-48 \mathrm{~h}^{-1}$. To ensure an infection probability of less than $1 \%$, a ventilation rate larger than common values $\left(100-350 \mathrm{~m}^{3} / \mathrm{h}\right.$ per infector and $1200-4000 \mathrm{~m}^{3} / \mathrm{h}$ per infector for $0.25 \mathrm{~h}$ and $3 \mathrm{~h}$ of exposure, respectively) is required. If the infector and susceptible person wear masks, then the ventilation rate ensuring a less than $1 \%$ infection probability can be reduced to a quarter respectively, which is easier to achieve by the normal ventilation mode applied in typical scenarios, including offices, classrooms, buses, and aircraft cabins. Strict preventive measures (e.g., wearing masks and preventing asymptomatic infectors from entering public spaces using tests) that have been widely adopted should be effective in reducing the risk of infection in confined spaces.
\end{abstract}

\author{
Keywords \\ COVID-19, \\ SARS-CoV-2, \\ ventilation, \\ infection probability, \\ Wells-Riley equation \\ Article History \\ Received: 11 June 2020 \\ Revised: 27 July 2020 \\ Accepted: 27 July 2020 \\ (c) Tsinghua University Press and \\ Springer-Verlag GmbH Germany, \\ part of Springer Nature 2020
}

\section{Introduction}

The once-in-a-century coronavirus disease 2019 (COVID-19) pandemic has shown that the airborne transmission of severe acute respiratory syndrome coronavirus 2 (SARS-CoV-2) cannot be ignored (Morawska and Cao 2020). Although the transmission of COVID-19 occurs mainly via droplets during close contact or via contaminated surfaces, a recent study showed that SARS-CoV-2 remains viable in aerosols for multiple hours (Van Doremalen et al. 2020). A recent report suggested that current research supports the possibility that SARS-CoV-2 can be spread via bioaerosols generated directly by patients' exhalation (National Research Council 2020). Furthermore, recent visualization studies also support that SARS-CoV-2 may spread between the infector and susceptible person simply by speaking (Anfinrud et al. 2020; Stadnytskyi et al. 2020). A recent field sampling study further indicated that SARS-CoV-2 is widely distributed in the air, and the transmission distance in the air might be up to $4 \mathrm{~m}$ (Guo et al. 2020). These results partly explain the easier spread of SARS-CoV-2 in confined spaces. The Wells-Riley equation is a classic model for quantifying the risk associated with airborne transmission of respiratory diseases (Riley et al. 1978). It has been used to demonstrate that some building factors (particularly the ventilation rate) are important removal mechanisms for airborne infectious agents (Escombe et al. 2007). In addition, a report by WHO suggested that insufficient ventilation increases disease transmission (Chartier et al. 2009). Therefore, ensuring a sufficient ventilation rate for offices, classrooms, and public transport is essential to reduce the potential risk of infection in these confined spaces, which is extremely important for daily life.

In this study, we employed the Wells-Riley equation to estimate the association between the infection probability and ventilation rate, where the quantum generation rate $(q)$ is a critical parameter. The value of $q$ of a COVID-19 infector is currently not well established, and we obtained this value based on its fitted correlation with the basic reproductive number $\left(R_{0}\right)$. We then estimated the association between

E-mail: binzhao@tsinghua.edu.cn 
the infection probability and ventilation rate for some typical scenarios, including offices, classrooms, buses, and aircraft cabins.

\section{Methods}

\subsection{Wells-Riley equation}

The Wells-Riley equation is as follows (Riley et al. 1978):

$P=\frac{C}{S}=1-\mathrm{e}^{-I q p t / Q}$

where $P$ is the probability of infection risk; $C$ is the number of cases that develop infection; $S$ is the number of susceptible people. $I$ is the number of source patients (infectors); in this study, we focused on normal scenarios (not health care settings for COVID-19 patients), thus, we assumed $I=1 . p$ is the pulmonary ventilation rate of each susceptible person per hour $\left(\mathrm{m}^{3} / \mathrm{h}\right) ; p=0.3 \mathrm{~m}^{3} / \mathrm{h}$ when people are sitting or participating in light activity indoors (Duan et al. 2013). $Q$ is the room ventilation rate $\left(\mathrm{m}^{3} / \mathrm{h}\right) ; q$ is the quantum generation rate produced by one infector $\left(h^{-1}\right) ; t$ is the exposure time (h).

The unit "quantum" in this risk model is not an actual physical unit; it is a hypothetical infectious dose unit that is typically back-calculated from epidemiological studies. One quantum is defined as a collection of pathogen particles that can infect susceptible people (Riley et al. 1978).

The Wells-Riley equation has two key assumptions, as follows: (1) droplet nuclei are evenly distributed in space, which means that the infection risk predicted by this equation is uniform within the space and (2) the equation neglects the viability and infectivity of the pathogen quanta.

Owing to the filtration effect of the mask, we considered that wearing a mask of infector can dilute exhaled pathogen concentration; while for the susceptible person, it's equivalent to increasing the room ventilation rate. The filtration efficiency of ordinary medical surgical masks on virus-laden aerosols is about 60\% (Hui et al. 2012), which can be set as $50 \%$ considering the influence of air leakage (Davies et al. 2013). The modified Wells-Riley equation is as follows:

$P=\frac{C}{S}=1-\mathrm{e}^{-I q p t\left(1-\eta_{\mathrm{I}}\right)\left(1-\eta_{\mathrm{s}}\right) / Q}$

where $\eta_{\mathrm{I}}$ is the exhalation filtration efficiency, $\eta_{\mathrm{I}}=50 \%$; $\eta_{\mathrm{s}}$ is the respiration filtration efficiency, $\eta_{s}=50 \%$.

Furthermore, a previous study modified the Wells-Riley equation to include deposition rates $\left(k_{\text {deposition }}\right)$ and filtration removal rates $\left(k_{\text {filtration }}\right)$ (Stephens 2012).

The $k_{\text {deposition }}$ depends primarily on the particle size, density, and room characteristics, such as air speeds and surface areas
(Fennelly and Nardell 1998; Fisk et al. 2004). It's measured (Liu et al. 2020b) that viral RNA in aerosols and found that SARS-CoV-2 aerosol mainly resides in two size ranges, namely $0.25-0.50 \mu \mathrm{m}$ and $0.50-1.00 \mu \mathrm{m}$. It's indicated that the range of $k_{\text {deposition }}$ is $0.21-0.63 \mathrm{~h}^{-1}$ with a mean value of $0.36 \mathrm{~h}^{-1}$ for aerosols in such two size ranges (Ji and Zhao 2015). This $k_{\text {deposition }}$ value has a negligible effect compared with the removal effect of ventilation in this study.

Regarding the $k_{\text {filtration }}$ value, if we treat the ventilation rate $(Q)$ as the equivalent clean air delivery rate (CADR), which is equal to the actual ventilation rate multiplied by filtration efficiency (Foarde 1999), then no modification is required to incorporate it.

Finally, the key input parameter when applying the Wells-Riley equation is $q$.

\subsection{Quantum generation rate with a reproductive number-based fitting approach}

So far, there have been no available reported values of $q$ of SARS-CoV-2. To obtain a reasonable $q$ value, we collected the known $q$ value and $R_{0}$ for other airborne transmitted infectious diseases in previous studies, and fitted the association between them. $R_{0}$ is the key epidemiological determinant that characterizes the transmission potential of an infectious disease, which is defined as the average number of infectious individuals created by a single infector in a susceptible population (Anderson et al. 1992). As both the $q$ and $R_{0}$ essentially determine the rate of spread of an epidemic and are estimated based on actual confirmed cases, we think that it may be logical to obtain the $q$ value based on its association with $R_{0}$ as a compromised but relatively reasonable approach.

We then estimated $q$ with the $R_{0}$ of COVID-19 based on the fitted equation. Table 1 lists the values of $q$ and $R_{0}$ for several typical infectious diseases collected from the references. It's reported that the transmission of respiratory pathogens such as measles, tuberculosis, middle east respiratory syndrome coronavirus (MERS), severe acute respiratory syndrome (SARS) and influenza in indoor environments are governed by similar physical and biological

Table 1 Quantum generation rate $(q)$ and basic reproductive number $\left(R_{0}\right)$ values of airborne transmitted infectious diseases

\begin{tabular}{lccc}
\hline & $q\left(\mathrm{~h}^{-1}\right)$ & $R_{0}$ & Reference \\
\hline Tuberculosis & $1-50$ & $2.22-5.46$ & Stephens 2012; Li et al. 2018 \\
MERS & $6-140$ & $0.50-1.20$ & WHO 2019 \\
SARS & $10-300$ & $2.00-5.00$ & WHO 2003 \\
Influenza & $15-500$ & $1.60-3.00$ & Lee et al. 2012 \\
Measles & $570-5600$ & $11.00-18.00$ & Plans Rubió 2012 \\
\hline
\end{tabular}


processes to COVID-19 (Stephens 2012; WHO 2003, 2019). With the values listed in this table as inputs, we fitted the association between $q$ and $R_{0}$ with a least square method implemented in Origin 2019.

\section{Results}

3.1 Fitted quantum generation rate of COVID-19 with the basic reproductive number

The fitted curve representing the association between $q$ and $R_{0}$ is shown in Figure 1, and the fitted equation is as follows:

$q=A+B_{1} \times R_{0}+B_{2} \times R_{0}^{2}$

where $A=-30.27958 \pm 26.07, B_{1}=-44.81536 \pm 12.34048$, and $B_{2}=19.67934 \pm 0.62317$.

With the widely used range of $R_{0}$ of 2.0-2.5 (Imai et al. 2020; Li et al. 2020; Majumder and Mandl 2020; Read et al. 2020; Zhao et al. 2020b), we obtained the corresponding range of $q$ for COVID-19, which was $14-48 \mathrm{~h}^{-1}$.

Considering that the $R_{0}$ of measles is much larger than that of other infectious diseases, we fitted the association between $q$ and $R_{0}$ with the same method but removed the $R_{0}$ of measles (Figure 2). The corresponding range of $q$ for COVID-19 is $2-40 \mathrm{~h}^{-1}$, which is comparable to the results obtained from Figure 1 and Eq. (3).

During the preparation of this manuscript, we found a study conducted by Lu et al. (2020) reporting a suspected case of airborne transmission of COVID-19 in Guangzhou, China, which is a suitable epidemiological case for the backcalculation of $q$. According to the description presented in the article, we back-calculated the value of $q$ to be $22 \mathrm{~h}^{-1}$. Furthermore, we also noticed another novel method for predicting the value of $q$ on the basis of the emitted viral load from the mouth (Buonanno et al. 2020). The results show that $q$ varies significantly as a function of different parameters

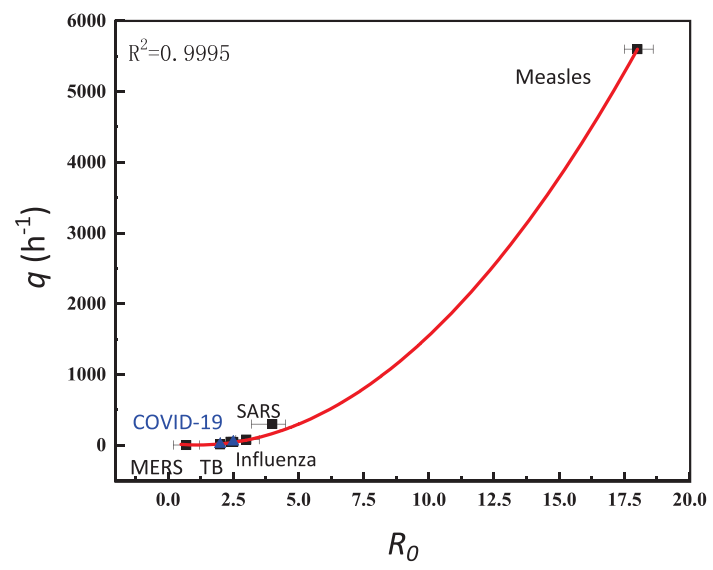

Fig. 1 The fitted curve between the quantum generation rate $(q)$ and basic reproductive number $\left(R_{0}\right)$ (with measles values)

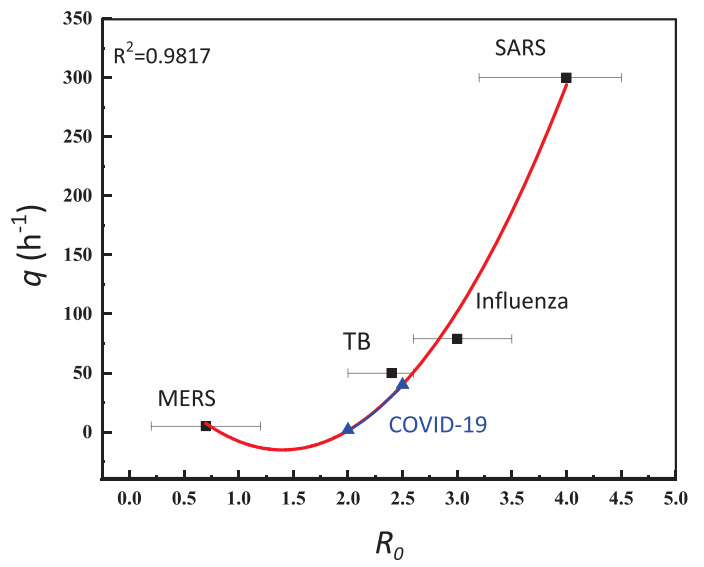

Fig. 2 The fitted curve between the quantum generation rate $(q)$ and basic reproductive number $\left(R_{0}\right)$ (without measles values)

(including inhalation rate, type of respiratory activity, and activity level). Our aim is to analyze the asymptomatic case in light exercise activity, which represents the typical characteristic of infectors in common public spaces. Considering this point, the corresponding $q$ reported by Buonanno et al. (2020) is between 10.5 and $33.9 \mathrm{~h}^{-1}$. Such values are in the same magnitude as the results in this study.

Based on the above analysis, it can be considered that the initially estimated value of $q$ in this study is reliable. Thus, we applied a $q$ value of $14-48 \mathrm{~h}^{-1}$ for the sedentary state for later analysis.

\subsection{Association between infection probability and ventilation rate}

\subsubsection{Infection probability}

Figure 3 shows the estimated association between the infection probability and ventilation rate. For the case with one infector inside of a confined space, a ventilation rate of $100-350 \mathrm{~m}^{3} / \mathrm{h}$ per infector was required to ensure an infection probability of less than $1 \%$ for $0.25 \mathrm{~h}$ of exposure of a susceptible person. A ventilation rate of $1200-4000 \mathrm{~m}^{3} / \mathrm{h}$ per infector was required to ensure an infection probability of less than $1 \%$ for $3 \mathrm{~h}$ of exposure of a susceptible person.

\subsubsection{Effect of wearing a mask}

The estimated association between the infection probability and ventilation rate for the cases with masks is shown in Figure 4. The results indicated that wearing a mask plays an important role in reducing the infection risk. For the case where there was one infector inside of a confined space, if both the infector and susceptible person wore masks, then the required ventilation rate to ensure an infection probability of less than $1 \%$ was reduced to $30-90 \mathrm{~m}^{3} / \mathrm{h}$ per infector for $0.25 \mathrm{~h}$ of exposure and $300-1000 \mathrm{~m}^{3} / \mathrm{h}$ per infector for $3 \mathrm{~h}$ of exposure of a susceptible person, respectively. 




(a)

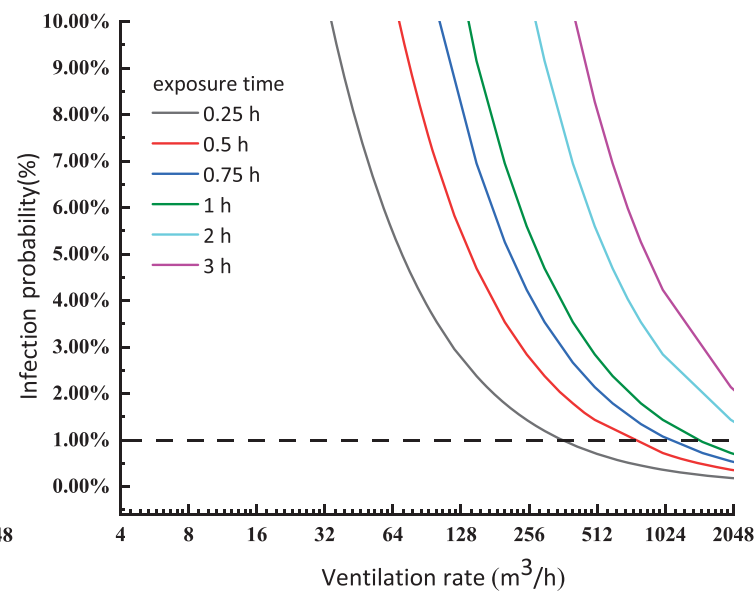

(b)

Fig. 3 Infection probability with different ventilation rates during different exposure times (without masks, one infector inside): (a) $q=14 \mathrm{~h}^{-1}$; (b) $q=48 \mathrm{~h}^{-1}$

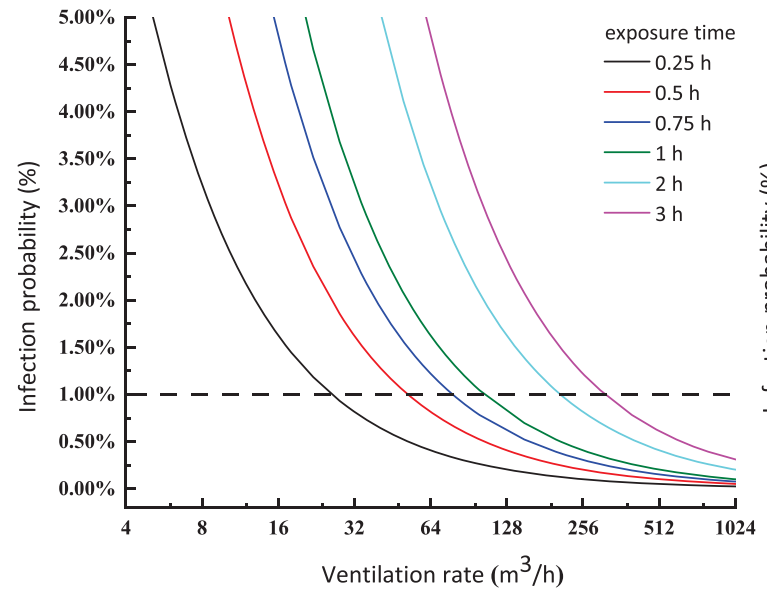

(a)

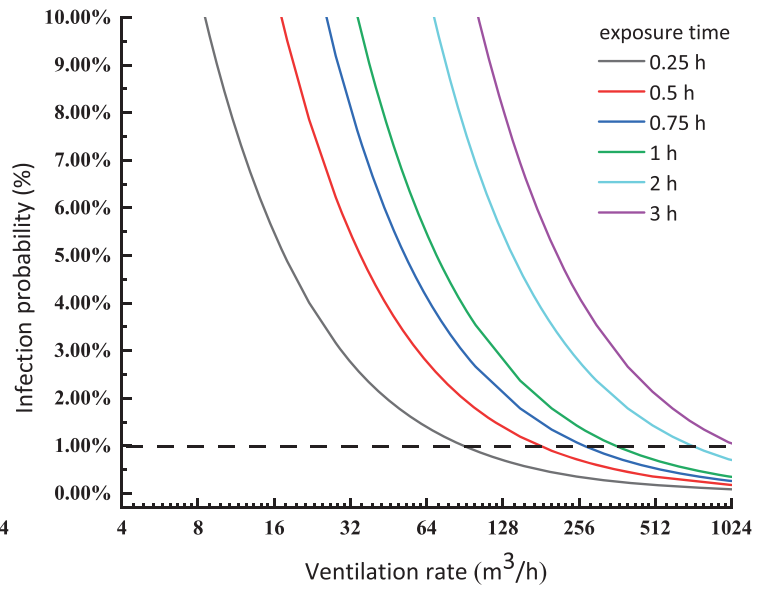

(b)

Fig. 4 Infection probability with different ventilation rates during different exposure times (with masks, one infector inside): (a) $q=14 \mathrm{~h}^{-1}$; (b) $q=48 \mathrm{~h}^{-1}$

\subsubsection{Typical scenarios}

Figure 5 shows the association of the estimated infection

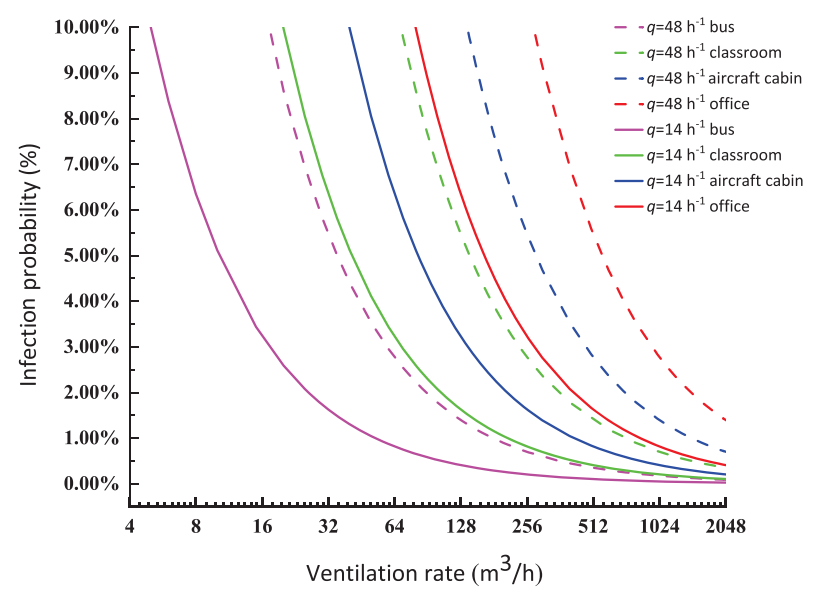

Fig. 5 Association between the infection probability and ventilation rate in typical scenarios (one infector inside) probability with the ventilation rate for some typical scenarios. Table 2 lists the corresponding air change rates (ACHs) for typical volumes of the examined confined spaces. The ACH is a measurement of how much fresh/clean air replaces indoor air in $1 \mathrm{~h}$ (Sherman and Wilson 1986), which is more widely used in engineering to determine if ventilation and air conditioning systems can provide a sufficient ventilation rate to ensure a low infection probability.

If people are wearing masks, then natural ventilation or normal mechanical ventilation can provide a sufficient ventilation rate to ensure that the infection probability is less than $1 \%$ for most scenarios (except for offices; Table 2). However, not wearing masks may result in a relatively higher infection risk for most cases, especially if $q$ reaches the maximum value. In these scenarios, additional measures could be suggested if the actual ventilation rate is out of a safe range, including UV sterilization, HEPA filtration and increasing outdoor air rate (Zhao et al. 2020a). 
Table 2 Air change rate (ACH) vs. infection probability (Y: with masks; N: without masks, one infector inside)

\begin{tabular}{|c|c|c|c|c|c|c|c|c|c|}
\hline \multirow{5}{*}{$\begin{array}{l}\text { Infection } \\
\text { probability }\end{array}$} & Scenario & \multicolumn{2}{|c|}{ Bus } & \multicolumn{2}{|c|}{ Classroom } & \multicolumn{2}{|c|}{ Aircraft cabin } & \multicolumn{2}{|c|}{ Office } \\
\hline & Volume $\left(\mathrm{m}^{3}\right)$ & \multicolumn{2}{|c|}{75} & \multicolumn{2}{|c|}{348} & \multicolumn{2}{|c|}{100} & \multicolumn{2}{|c|}{150} \\
\hline & Exposure time (h) & \multicolumn{2}{|c|}{0.5} & \multicolumn{2}{|c|}{2.0} & \multicolumn{2}{|c|}{4.0} & \multicolumn{2}{|c|}{8.0} \\
\hline & & \multicolumn{8}{|c|}{$\mathrm{ACH}\left(\mathrm{h}^{-1}\right)$} \\
\hline & & $\mathrm{Y}$ & $\mathrm{N}$ & $\mathrm{Y}$ & $\mathrm{N}$ & $\mathrm{Y}$ & $\mathrm{N}$ & $\mathrm{Y}$ & $\mathrm{N}$ \\
\hline $2.0 \%$ & & 0.33 & 1.30 & 0.28 & 1.15 & 2.00 & 8.00 & 2.70 & 10.00 \\
\hline $1.5 \%$ & & 0.48 & 1.80 & 0.40 & 1.70 & 3.00 & 11.00 & 3.30 & 14.00 \\
\hline $1.0 \%$ & $q=14 \mathrm{~h}^{-1}$ & 0.70 & 2.80 & 0.60 & 2.40 & 4.00 & 16.00 & 6.00 & 20.00 \\
\hline $0.5 \%$ & & 1.40 & 5.60 & 1.20 & 4.80 & 9.00 & 33.00 & 10.00 & 43.00 \\
\hline $0.1 \%$ & & 7.00 & 27.00 & 5.50 & 20.00 & 35.00 & 160.00 & 50.00 & 200.00 \\
\hline $2.0 \%$ & & 1.20 & 4.80 & 1.00 & 4.00 & 7.00 & 30.00 & 10.00 & 36.00 \\
\hline $1.5 \%$ & & 1.60 & 6.40 & 1.40 & 5.00 & 9.00 & 38.00 & 12.00 & 53.00 \\
\hline $1.0 \%$ & $q=48 \mathrm{~h}^{-1}$ & 2.40 & 9.60 & 2.00 & 7.00 & 15.00 & 55.00 & 18.00 & 80.00 \\
\hline $0.5 \%$ & & 4.80 & 19.00 & 3.50 & 15.00 & 29.00 & 110.00 & 33.00 & 133.00 \\
\hline $0.1 \%$ & & 24.00 & 93.00 & 20.00 & 71.00 & 125.00 & 550.00 & 200.00 & 666.00 \\
\hline
\end{tabular}

${ }^{*}$ The ACHs in red text indicate that the ventilation rates could be achieved with a normal ventilation system or natural ventilation for the corresponding scenarios.

\section{Discussion}

$R_{0}$ varies due to different methodological issues, including the utilized datasets, confirmed cases, outbreaking region and mathematical models. Several methods have been produced, resulting in different estimations of $R_{0}$. For example, there are studies showing that the $R_{0}$ of COVID-19 can be reached a high level of 6.47 (Tang et al. 2020). However, $R_{0}$ seems to be stabilized at around 2-3, which is obtained from a large database (Liu et al. 2020a) and from more different researches (D’Arienzo and Coniglio 2020; Imai et al. 2020; Li et al. 2020; Majumder and Mandl 2020; Read et al. 2020; Riou and Althaus 2020; Zhao et al. 2020b), the data used includes both the Mainland of China and overseas outbreak up to March, 2020. Thus the $R_{0}$ of 2-2.5 here can be expected to be reliable, as it builds upon more case data.

Given the large uncertainties associated with each of the parameters, we relied on two assumptions in an attempt to link the indoor ventilation strategy to infectious disease risk models, namely, the ventilation efficiency was assumed to be 1 and the concentration of viruses was assumed to be homogeneous in the indoor environment.

The estimation indicates that when an infector remains in a public confined space, the infection risk is relatively high with an infection probability of approximately $2 \%$ at the common ventilation rate $\left(500-2500 \mathrm{~m}^{3} / \mathrm{h}\right.$ per infector for $0.25 \mathrm{~h}$ of exposure; Figure 3). Such findings may partly explain the early large-scale epidemics in China and European and American countries.

Thus, preventing the infectors (especially asymptomatic infectors that have not been confirmed) from entering these types of public spaces is critical to suppress the spread of the virus via airborne transmission. Current measures, strict screening of asymptomatic infectors with wider nucleic acid tests, and home isolation have already ensured that infectors are unlikely to enter public spaces and have ensured a relatively safe environment in confined spaces.

Furthermore, the above findings imply that wearing an ordinary medical surgical mask is effective; thus, it is important to advise people to wear masks when they enter or remain in confined spaces.

It is acknowledged that COVID-19 is transmitted mainly by close contact or droplets deposited on contaminated surfaces. However, the risk of potential or opportunistic airborne transmission cannot be ignored, especially in public confined spaces. The value of $q$ also warrants further study. Furthermore, strict preventive measures have been widely adopted, such measure provides a prerequisite for common ventilation strategies to ensure a sufficiently low risk of airborne transmitted infection.

\section{Conclusion}

This study employed Wells-Riley equation to predict infection probability in some typical scenarios based on the estimated quantum generation rate. The main conclusions are as follows:

(1) A quantum generation rate of $14-48 \mathrm{~h}^{-1}$ for COVID-19 is obtained with a reproductive number-based fitting approach.

(2) The Infection probability less than $1 \%$ requires ventilation rate larger than $100-350 \mathrm{~m}^{3} / \mathrm{h}$ per infector and $1200-$ $4000 \mathrm{~m}^{3} / \mathrm{h}$ per infector for $0.25 \mathrm{~h}$ and $3 \mathrm{~h}$ of exposure.

(3) Wearing an ordinary medical surgical mask is effective to reduce the infection risk. 


\section{Acknowledgements}

The study was supported by the Ministry of Science and Technology of China (No. 2020YFC0842500) and the National Natural Science Foundation of China (No. 52041602, No. 51521005).

\section{References}

Anderson RM, Anderson B, May RM (1992). Infectious Diseases of Humans: Dynamics and Control. Oxford, UK: Oxford University Press.

Anfinrud P, Stadnytskyi V, Bax CE, Bax A (2020). Visualizing speechgenerated oral fluid droplets with laser light scattering. New England Journal of Medicine, 382: 2061-2063.

Buonanno G, Stabile L, Morawska L (2020). Estimation of airborne viral emission: Quanta emission rate of SARS-CoV-2 for infection risk assessment. Environment International, 141: 105794.

Chartier Y, Atkinson J, Pessoa-Silva C (2009). Natural ventilation for infection control in health-care settings. World Health Organization.

D'Arienzo M, Coniglio A (2020). Assessment of the SARS-CoV-2 basic reproduction number, R0, based on the early phase of COVID-19 outbreak in Italy. Biosafety and Health, 2: 57-59.

Davies A, Thompson KA, Giri K, Kafatos G, Walker J, Bennett A (2013). Testing the efficacy of homemade masks: would they protect in an influenza pandemic? Disaster Medicine and Public Health Preparedness, 7: 413-418.

Duan X, Zhao X, Wang B, Chen Y, Cao S (2013). Exposure Factors Handbook of Chinese Population (Adults). Beijing: China Environmental Science Press. (in Chinese)

Escombe AR, Oeser CC, Gilman RH, Navincopa M, Ticona E, et al. (2007). Natural ventilation for the prevention of airborne contagion. PLoS Medicine, 4: e68.

Fennelly KP, Nardell EA (1998). The relative efficacy of respirators and room ventilation in preventing occupational tuberculosis. Infection Control and Hospital Epidemiology, 19: 754-759.

Fisk WJ, Seppanen O, Faulkner D, Huang J (2004). Economic benefits of an economizer system: Energy savings and reduced sick leave. Technical Report, LBNL-54475, Lawrence Berkeley National Laboratory.

Foarde KK (1999). Methodology to perform clean air delivery rate type determinations with microbiological aerosols. Aerosol Science and Technology, 30: 235-245.

Guo Z-D, Wang Z-Y, Zhang S-F, Li X, Li L, et al. (2020). Aerosol and surface distribution of severe acute respiratory syndrome coronavirus 2 in hospital wards, Wuhan, China, 2020. Emerging Infectious Diseases, 26: 1583-1591.

Hui DS, Chow BK, Chu L, Ng SS, Lee N, et al. (2012). Exhaled air dispersion during coughing with and without wearing a surgical or N95 mask. PLoS One, 7: e50845.

Imai N, Cori A, Dorigatti I, Baguelin M, Donnelly CA, et al. (2020). Report 3: Transmissibility of 2019-nCoV. Imperial College London. Available at http://hdl.handle.net/10044/1/77148.
Ji W, Zhao B (2015). Contribution of outdoor-originating particles, indoor-emitted particles and indoor secondary organic aerosol (SOA) to residential indoor $\mathrm{PM}_{2.5}$ concentration: A model-based estimation. Building and Environment, 90: 196-205.

Lee S, Golinski M, Chowell G (2012). Modeling optimal age-specific vaccination strategies against pandemic influenza. Bulletin of Mathematical Biology, 74: 958-980.

Li HL, Zhang XL, Wang K (2018). A quantitative study on the epidemic situation of tuberculosis based on the transmission disease dynamics in 14 prefectures of Xinjiang from 2005 to 2017. Chinese Journal of Infection Control, 17(11): 945-950. (in Chinese)

Li Q, Guan X, Wu P, Wang X, Zhou L, et al. (2020). Early transmission dynamics in Wuhan, China, of novel coronavirus-infected pneumonia. New England Journal of Medicine, 382: 1199-1207.

Liu Y, Gayle AA, Wilder-Smith A, Rocklöv J (2020a). The reproductive number of COVID-19 is higher compared to SARS coronavirus. Journal of Travel Medicine, 27(2): taaa021. https://doi.org/10.1093/ jtm/taaa021

Liu Y, Ning Z, Chen Y, Guo M, Liu Y, et al. (2020b). Aerodynamic analysis of SARS-CoV-2 in two Wuhan hospitals. Nature, 582: 557-560.

Lu J, Gu J, Li K, Xu C, Su W, et al. (2020). COVID-19 outbreak associated with air conditioning in restaurant, Guangzhou, China, 2020. Emerging Infectious Diseases, 26: 1628-1631.

Majumder M, Mandl KD (2020). Early transmissibility assessment of a novel coronavirus in Wuhan, China. SSRN Electronic Journal, https://doi.org/10.2139/ssrn.3524675.

Morawska L, Cao J (2020). Airborne transmission of SARS-CoV-2: The world should face the reality. Environment International, 139: 105730.

National Research Council (2020). Rapid Expert Consultation on the Possibility of Bioaerosol Spread of SARS-CoV-2 for the COVID-19 Pandemic. Washington DC: The National Academies Press.

Plans Rubió P (2012). Is the basic reproductive number $\left(R_{0}\right)$ for measles viruses observed in recent outbreaks lower than in the pre-vaccination era? Euro Surveillance, 17(31): 20233.

Read JM, Bridgen JRE, Cummings DAT, Ho A, Jewell CP (2020). Novel coronavirus 2019-nCoV: Early estimation of epidemiological parameters and epidemic predictions. medRxiv, https://doi.org/ 10.1101/2020.01.23.20018549.

Riley EC, Murphy G, Riley RL (1978). Airborne spread of measles in a suburban elementary school. American Journal of Epidemiology, 107: 421-432.

Riou J, Althaus CL (2020). Pattern of early human-to-human transmission of Wuhan 2019 novel coronavirus (2019-nCoV), December 2019 to January 2020. Euro Surveillance, 25: 2000058.

Sherman MH, Wilson DJ (1986). Relating actual and effective ventilation in determining indoor air quality. Building and Environment, 21 : 135-144.

Stadnytskyi V, Bax CE, Bax A, Anfinrud P (2020). The airborne lifetime of small speech droplets and their potential importance in SARSCoV-2 transmission. Proceedings of the National Academy of Sciences of the United States of America(PNAS), 117: 11875-11877.

Stephens B (2012). HVAC filtration and the Wells-Riley approach to assessing risks of infectious airborne diseases. National Air Filtration Association (NAFA) Foundation Report. 
Tang B, Bragazzi NL, Li Q, Tang S, Xiao Y, Wu J (2020). An updated estimation of the risk of transmission of the novel coronavirus (2019-nCov). Infectious Disease Modelling, 5: 248-255.

Van Doremalen N, Bushmaker T, Morris DH, Holbrook MG, Gamble A, et al. (2020). Aerosol and surface stability of SARS-CoV-2 as compared with SARS-CoV-1. New England Journal of Medicine, 382: $1564-1567$.

WHO (2003). Consensus document on the epidemiology of severe acute respiratory syndrome (SARS) (No. WHO/CDS/CSR/GAR/ 2003.11). World Health Organization.
WHO (2019). WHO MERS global summary and assessment of risk, July 2019 (No. WHO/MERS/RA/19.1). World Health Organization.

Zhao B, Liu Y, Chen C (2020a). Air purifiers: A supplementary measure to remove airborne SARS-CoV-2. Building and Environment, 177: 106918.

Zhao S, Lin Q, Ran J, Musa SS, Yang G, et al. (2020b). Preliminary estimation of the basic reproduction number of novel coronavirus (2019-nCoV) in China, from 2019 to 2020: A data-driven analysis in the early phase of the outbreak. International Journal of Infectious Diseases, 92: 214-217. 